\title{
Spin-Charge Separation, Anomalous Scaling and the Coherence of Hopping in exactly solved Two Chain Models
}

\author{
Nic Shannon, Yanmin Li and Nicholas d'Ambrumenil \\ Department of Physics, University of Warwick, Coventry, England
}

(February 10, 2018)

\begin{abstract}
The coherence of transport between two one-dimensional interacting Fermi liquids, coupled by single particle hopping and interchain interaction, is examined in the context of two exactly soluble models. It is found that the coherence of the inter-chain hopping depends on the interplay between interchain hopping and inter-chain interaction terms, and not simply on the ground state spectral properties of an isolated chain. Specifically, the splitting of levels in associated with interchain hopping in a $g_{4}$ soluble model is found to be enhanced by the introduction of interchain interaction. It is also shown that, for an exactly solvable model with both $g_{2}$ and $g_{4}$ interactions, coherent interchain hopping coexists with anomalous scaling and non-Fermi liquid behavior in the chain direction.
\end{abstract}

PACS Nos. 71.27.+a, 05.30.Fk, 71.10.+x

Typeset using REVTEX 


\section{INTRODUCTION}

Although the physics of an ideal one-dimensional Fermi gas has been widely and profitably studied for more than twenty years [1,2], our understanding of real low-dimensional materials is still limited by the lack of a clear picture of how the crossover in electronic properties between strictly one-dimensional and three-dimensional limits occurs. While it is clear that a one-dimensional interacting Fermi gas cannot be a Landau Fermi Liquid, the possibility of finding in higher dimensions a ground state analogous to the non-Fermi Liquid (NFL) ground states found in one dimension (in particular the Luttinger Liquid (LL), characterized by different velocities for spin and charge excitations, and an anomalous exponent $\alpha$ controlling spectral properties) is still a subject of much debate. Interest in these issues has been stimulated by the unusual properties found in high temperature superconductors and the wide variety of other material in which the electrons are believed to be both strongly correlated and confined to move in low dimension.

Theoretical consideration of two dimensional interacting Fermi liquids with fully two dimensional Fermi surfaces by a number of authors and techniques [3, 4] has consistently found that the Landau Fermi Liquid state $i s$ stable, excepting cases of extremely long range interaction which are of questionable physical relevance. Coupled chain models (generally comprising a large number of identical one dimensional interacting Fermi liquids coupled by weak interchain hopping) have also attracted much attention [5-15]. These are directly ap-

plicable to the large class of quasi one-dimensional conductors which are held to be strongly correlated (for example the organic spin density wave system $T T F-T C N Q$ ).

The effects which might be expected to modify or destroy the one-dimensional properties of an isolated chain are interchain hopping and interchain interaction. These are usually introduced as a single particle hopping between neighboring chains, and a density-density interaction between pairs of chains, with the possibility of backward scattering or umklapp terms in the interaction generally being neglected. While the problem of chains coupled by interaction alone is soluble by the technique of bosonization [15] 17], interchain hopping 
spoils the special current conservation laws (Ward identities) which make the solution of the problem by bosonization techniques possible. In this regard it is analogous to the onedimensional backward scattering problem [18].

Scaling theories (perturbative renormalization group techniques) have been applied most intensively to the problem of two chains coupled by interchain hopping and interaction [8.13,10], and have given considerable insight into the ground state instabilities (dominant fluctuations) found for different combinations of interchain and intrachain interaction. These models have also been studied numerically [11,12]. The simplest scaling arguments 13, 15 suggest that for spinless fermions, there is a critical value ( $\alpha=\frac{1}{2}$ for spinless fermions) of the anomalous exponent associated with the one-dimensional Luttinger liquid ground state, above which weak hopping is an irrelevant perturbation. Correlation of electrons on a single chain can then lead to their confinement within it. This value of $\alpha$ can only be regarded as placing a bound on the stability of the one-dimensional Luttinger liquid state. It does not imply that systems formed form chains with $0<\alpha<\frac{1}{2}$ automatically flow to a higher dimensional fixed point (i.e. Fermi Liquid); where interchain hopping is a relevant perturbation it is not possible to determine the ground state properties of the coupled chain system from scaling arguments alone. In fact the values of $\alpha$ considered applicable to real materials are often less than $1 / 2$. For example, it is known that for a one-dimensional Hubbard chain $\alpha \leq \frac{1}{8}$ in the limit $U \rightarrow \infty$.

The debate over the role played by interchain/interplane hopping in the stability of NFL states was extended by Anderson's suggestion [19] that weak single particle hopping between Luttinger Liquids cannot generate band structure in the direction of the hopping, even in cases where this hopping is relevant in the Renormalization Group Sense. Instead there is "confinement by decoherence": the system retains its non-Fermi Liquid (NFL) character in one (or two) directions, and suffers only diffusive motion of electrons perpendicular to this direction [20 22]. Since this method of confinement need not suppress Josephson Coupling (pair hopping) between NFL's, a set of weakly coupled NFL planes can become a threedimensional (anisotropic) superconductor, with c-axis transport properties matching those 
of the cuprates [23].

Following this suggestion, the question of whether arbitrarily weak interchain hopping $t_{\perp}$ can cause a splitting of energy levels (analogous to the formation of binding and antibinding bands in the free electron case) in interacting two chain models has come to be seen as a useful first step towards understanding whether coherent transport is possible in the physically relevant N-chain case. Two recent numerical studies [12,27] have found evidence of such a splitting in finite size systems. In the case of the spinless two chain model studied by Capponi et al. [27], the splitting $\Delta E$ due to interchain hopping may be directly observed in the electronic spectral function, and decreases with increasing $\alpha$ from the free electron value of $2 t_{\perp}$ (for $\left.\alpha=0\right)$. Finite size scaling of the results for $\Delta E(L) / 2 t_{\perp}$ as a function of $\alpha$, shows a finite but steadily decreasing splitting for all $\alpha<0.3$, compatible with the results of scaling arguments.

It is clear that, as the couplings between an array of chains embedded in two or three dimensions become stronger, the system becomes more nearly isotropic, and must eventually cross over to the two (three) dimensional limit in which the Fermi liquid description may be applied. This issue is complicated by the possibility of a crossover to an ordered twoor three-dimensional state, stabilized by many particle exchange between chains (as has been found in RG studies of two chain models [8, 13] ). The nature of this crossover was addressed by Anderson [19] and recently by Tsvelik [24], who identified the possibility of coherent transport between chains with the existence of a well-defined pole in the single particle Green's function.

In this paper we establish what may be said about the coherence of hopping between chains in two soluble extensions of one-dimensional models: a special case of the general two chain model displaying both spin charge separation and anomalous scaling, and an extension of the single branch spinful two chain model originally studied by Fabrizio and Tsvelik [25,26,28] to include interchain interaction. We will compare our results with existing numerical and perturbative analyses. We begin by briefly reviewing Anderson's criterion for coherence and its application to coupled free electron chains. 


\section{CRITERION FOR COHERENCE}

The arguments about coherence presented by Clarke, Strong and Anderson (CSA) are rooted in an analogy between the problem of hopping between Luttinger Liquids and that of a two level system (TLS) coupled to a dissipative ohmic background [29,30. In the light of this analogy, these authors propose that the criterion for coherence should be the probability of the system returning to a specially prepared state during its intermediate time evolution after the sudden introduction of hopping between chains at time $t=0$. The state in which the system is prepared is an eigenstate of the original Hamiltonian for $t<0$ in which one of the two chains carries additional charge. This corresponds to the TLS having a non-zero probability of being found in one of its two states. A fully coherent (e.g. free electron) system will evolve away from its original state, but return to it over a time scale set by the size of the hopping matrix element. An incoherent system will never return to its original state - in the language of the dissipative TLS, the probability of it being found in either state must relax to zero.

We may formally consider the probability $P(t)$ of the system returning to its initial state

$$
\begin{aligned}
P(t) & =|A(t)|^{2} \\
A(t) & =<\psi_{0}\left|e^{i H^{\prime} t} e^{-i H t}\right| \psi_{0}> \\
H^{\prime} & =H+H_{0 \perp} \\
H \mid \psi_{0}> & =E_{0} \mid \psi_{0}>.
\end{aligned}
$$

where $\left|\psi_{0}\right\rangle$ is a specially prepared eigenstate of $H$ with additional charge $\Delta N$ on one of the chains, and $H_{0 \perp}$ is an interchain hopping term, generally taken to be of the form

$$
H_{0 \perp}=-t_{\perp} \sum_{k, \sigma}\left[c_{\sigma}^{(1) \dagger}(k) c_{\sigma}^{(2)}(k)+c_{\sigma}^{(2) \dagger}(k) c_{\sigma}^{(1)}(k)\right]
$$

For free electron chains we have

$$
H=H_{0 \|}=\sum_{k, \sigma} \epsilon(k)\left[c_{\sigma}^{(1) \dagger}(k) c_{\sigma}^{(1)}(k)+c_{\sigma}^{(2) \dagger}(k) c_{\sigma}^{(2)}(k)\right]
$$


and the overlap $A(t)$ may be evaluated exactly; the system can be treated as a product of two level (chain) systems for each wave number $k$ along the chains, and the Hamiltonian for each wave number (and spin index) separately diagonalized. The occupation of states labeled by $k$, which are initially occupied on one chain but not on the other, then oscillates between chains with frequency $2 t_{\perp}$, so that in the presence in the initial state of extra charge $\Delta N$ on one chain, we find [31]

$$
P(t)=\cos ^{2 \Delta N}\left(t_{\perp} t\right) .
$$

More generally, we may expand $\mid \psi_{0}>$ in the eigenstates of $H^{\prime}$

$$
\begin{aligned}
\mid \psi_{0}> & =\sum_{n} c_{n} \mid \phi_{n}> \\
H^{\prime} \mid \phi_{n}> & =\epsilon_{n} \mid \phi_{n}> \\
A(t) & =\sum_{n, m} c_{m}^{*} c_{n}<\phi_{m}\left|e^{-i H^{\prime} t}\right| \phi_{n}>e^{i E_{0}} \\
& =\sum_{n}\left|c_{n}\right|^{2} e^{i\left(E_{0}-E_{n}\right) t}
\end{aligned}
$$

Our model is not dissipative, and the quantity $P(t)$ possesses full time reversal symmetry, but the overlap $A(t)$ can display damping due to the interference between modes of different frequency $E_{n}$. In finite size systems, where one might expect $P(t)$ eventually to return to one, damping can still be observed over all physical time-scales [32].

In order to extend their macroscopic analysis to correlated systems, CSA study the rate of decay of the system from its original state after the introduction of hopping using Fermi's Golden rule. They claim that an anomalous exponent $\alpha>\frac{1}{4}$ is sufficient to impose a lower bound $t_{\perp}^{c}$ on the value of $t_{\perp}$ needed to generate coherent interchain hopping, so for Luttinger Liquids with $\alpha$ exceeding $\frac{1}{4}$, arbitrarily weak interliquid hopping cannot generate band structure [20 22].

Mila and Poilblanc have calculated the Fourier transform of the overlap $A(t)$ numerically for a number of $2 \times L \mathrm{t}$-J Ladders [33], and find that the coherence demonstrated by this quantity in the limit $t_{\perp} \rightarrow 0$ is not simply determined by the anomalous scaling parameter found in the spectral function for an isolated chain, as suggested in CSA, but is considerably 
enhanced near integrable points of the single chain t-J plane. They suggest that this may be understood in terms of the level statistics of the problem; for an integrable system the eigenstates with additional charge $\Delta N$ are highly degenerate. Degenerate perturbation theory must therefore be applied tending to yield a splitting in energy levels linear in $t_{\perp}$ and associated coherence. One could also say that level repulsion in a nonintegrable system will tend to lead to a bigger spread of frequencies entering the expression for the overlap $A(t)$, and so to a greater degree of damping in $P(t)$ 32].

We can also relate the behavior of $P(t)$ to conserved quantities within the problem. For free electrons $H=H_{0 \|}$ and, since $\left[H_{0 \|}, H_{0 \perp}\right]=0$ we may use the Baker-Hausdorf identity $e^{A+B}=e^{A} e^{B} e^{-\frac{1}{2}[A, B]}$ (where $\left.[A,[A, B]]=[B,[A, B]]=0\right)$ to combine the terms in $A(t)$, and show as above that

$$
P(t)=\left|<\psi_{0}\right| e^{i H_{0 \perp} t}\left|\psi_{0}>\right|^{2}=\left[\cos \left(t_{\perp} t\right)\right]^{2 \Delta N} .
$$

Making a change to binding/antibinding coordinates

$$
\begin{aligned}
& c_{\sigma}^{(1) \dagger}(k)=\frac{1}{\sqrt{2}}\left(b_{\sigma}^{\dagger}(k)+a_{\sigma}^{\dagger}(k)\right) \\
& c_{\sigma}^{(2) \dagger}(k)=\frac{1}{\sqrt{2}}\left(b_{\sigma}^{\dagger}(k)-a_{\sigma}^{\dagger}(k)\right)
\end{aligned}
$$

we see that the condition for complete coherence is that the Hamiltonian $H$ commute with

$$
H_{0 \perp}=-t_{\perp} \sum_{k, \sigma}\left[b_{\sigma}^{\dagger}(k) b_{\sigma}(k)-a_{\sigma}^{\dagger}(k) a_{\sigma}(k)\right]
$$

i.e. that the difference in the number of particles in the binding and antibinding bands be a good quantum number of the system; if the original Hamiltonian may be diagonalized in the basis of eigenstates of the perturbation then the original state will evolve completely coherently. The introduction of interaction can quickly be seen to spoil this property of the Hamiltonian. For example, for the two chain Hubbard Model

$$
\begin{aligned}
H & =H_{1}+H_{2}+H_{0 \perp} \\
H_{1} & =-t_{\|} \sum_{i, \sigma} \psi_{i+1 \sigma}^{\dagger(1)} \psi_{i \sigma}^{(1)} \psi_{i \sigma}^{\dagger(1)} \psi_{i+1 \sigma}^{(1)}+U \sum_{i, \sigma} n_{i, \sigma}^{(1)} n_{i-\sigma}^{(1)} \\
H_{0 \perp} & =-t_{\perp} \sum_{i \sigma} \psi_{i \sigma}^{\dagger(1)} \psi_{i \sigma}^{(2)}+\psi_{i \sigma}^{\dagger(2)} \psi_{i \sigma}^{(1)},
\end{aligned}
$$


giving

$$
\left[H_{0 \perp}, H\right]=-t_{\perp} U \sum_{i, \sigma} n_{i \sigma}^{(1)}\left[\psi_{i-\sigma}^{\dagger(1)} \psi_{i-\sigma}^{(2)}+\psi_{i-\sigma}^{\dagger(2)} \psi_{i-\sigma}^{(1)}\right]+n_{i \sigma}^{(2)}\left[\psi_{i-\sigma}^{\dagger(2)} \psi_{i-\sigma}^{(1)}+\psi_{i-\sigma}^{\dagger(1)} \psi_{i-\sigma}^{(2)}\right]
$$

Th quantity $\left[H_{\perp}, H\right]$ does not commute with either part of $H$, on account of the terms of the form $\psi_{i, \sigma}^{\dagger(b)} \psi_{i, \sigma}^{(a)} \psi_{i,-\sigma}^{\dagger(a)} \psi_{i,-\sigma}^{(b)}$ in the interaction, so complete coherence is lost. At a microscopic level, this need not mean that interchain hopping is entirely incoherent, only that $N^{(b)}-N^{(a)}$ is not a good quantum number for the system and that the interchain hopping has some incoherent part. We might expect the orthogonality of the interacting and noninteracting ground states of the 1D Fermi gas (measured by the parameter $\alpha$ ) to render interchain hopping highly incoherent, and so frustrate the formation of band structure in the interchain direction. In fact the issue is more subtle than this; the effect of the commutator (10) could be cancelled by the introduction of an additional interaction term $U \sum_{i, \sigma} n_{i, \sigma}^{(2)} n_{i-\sigma}^{(1)}$. This will not restore fermionic quasiparticle character to the electronic states of the individual chains in the absence of $H_{0 \perp}$, but will make possible the macroscopically coherent evolution of the specially prepared state described above. Thus we must either reject our macroscopic criterion for coherence described above, or accept that hopping between non-Fermi liquids can be entirely coherent. We draw the latter conclusion and illustrate this point in more detail for a continuum model in the following section.

\section{A SOLUBLE TWO CHAIN MODEL WITH ANOMALOUS DIMENSION AND COHERENT INTERCHAIN HOPPING}

We take as a general Hamiltonian for the coupled chain problem :

$$
H=H_{0}+V_{\|}+V_{\perp}
$$

Here,

$$
\begin{aligned}
H_{0} & =\sum_{k, \sigma, m} \epsilon_{L}(k)\left[c_{L \sigma}^{(1) \dagger}(k) c_{L \sigma}^{(1)}(k)+c_{L \sigma(k)}^{(2) \dagger} c_{L \sigma}^{(2)}(k)\right] \\
& -t_{\perp}\left[c_{L \sigma}^{(1) \dagger}(k) c_{L \sigma}^{(2)}(k)+c_{L \sigma}^{(2) \dagger}(k) c_{L \sigma}^{(1)}(k)\right]+[L \rightarrow R],
\end{aligned}
$$




$$
\begin{aligned}
& V_{\|}=\frac{1}{2 L} \sum_{q>0, \sigma, \sigma^{\prime}} g_{4 \|}\left[\rho_{L \sigma}^{(1)}(-q) \rho_{L \sigma^{\prime}}^{(1)}(q)+\rho_{L \sigma}^{(2)}(-q) \rho_{L \sigma^{\prime}}^{(2)}(q)\right] \\
& +g_{2 \|}\left[\rho_{L \sigma}^{(1)}(-q) \rho_{R \sigma^{\prime}}^{(1)}(q)+\rho_{L \sigma}^{(2)}(-q) \rho_{R \sigma^{\prime}}^{(2)}(q)\right] \\
& +g_{4 \|}\left[\left(N_{L \sigma}^{(1)}-N_{L 0 \sigma^{\prime}}^{(1)}\right)^{2}+\left(N_{L \sigma}^{(2)}-N_{L 0 \sigma^{\prime}}^{(2)}\right)^{2}\right] \\
& +g_{2 \|}\left[\left(N_{L \sigma}^{(1)}-N_{L 0 \sigma}^{(1)}\right)\left(N_{R \sigma^{\prime}}^{(1)}-N_{R 0 \sigma^{\prime}}^{(1)}\right)+\left(N_{L \sigma}^{(2)}-N_{L 0 \sigma}^{(2)}\right)\left(N_{R \sigma^{\prime}}^{(2)}-N_{R 0 \sigma^{\prime}}^{(2)}\right]\right. \\
& +[L \rightarrow R, q \rightarrow-q] \\
& V_{\perp}=\frac{1}{2 L} \sum_{q>0, \sigma, \sigma^{\prime}} g_{4 \perp}\left[\rho_{L \sigma}^{(1)}(-q) \rho_{L \sigma^{\prime}}^{(2)}(q)+\rho_{L \sigma}^{(2)}(-q) \rho_{L \sigma^{\prime}}^{(1)}(q)\right] \\
& +g_{2 \perp}\left[\rho_{L \sigma}^{(1)} \rho_{R \sigma^{\prime}}^{(2)}(q)+\rho_{L \sigma}^{(2)}(-q) \rho_{R \sigma^{\prime}}^{(1)}(q)\right] \\
& +g_{4 \perp}\left[\left(N_{L \sigma}^{(1)}-N_{L 0 \sigma^{\prime}}^{(1)}\right)^{2}+\left(N_{L \sigma}^{(2)}-N_{L 0 \sigma^{\prime}}^{(2)}\right)^{2}\right] \\
& +g_{2 \perp}\left[\left(N_{L \sigma}^{(1)}-N_{L 0 \sigma}^{(1)}\right)\left(N_{R \sigma^{\prime}}^{(1)}-N_{R 0 \sigma^{\prime}}^{(1)}\right)+\left(N_{L \sigma}^{(2)}-N_{L 0 \sigma}^{(2)}\right)\left(N_{R \sigma^{\prime}}^{(2)} N_{R 0 \sigma^{\prime}}^{(2)}\right]\right. \\
& +[L \rightarrow R, q \rightarrow-q] \\
& \rho_{m \sigma}^{(i)}(q)=\sum_{p \sigma} c_{m \sigma}^{(i) \dagger}(p+q) c_{m \sigma}^{(i)}(p) \\
& N_{m \sigma}^{(i)}=\sum_{p \sigma} c_{m \sigma}^{(i) \dagger}(p) c_{m \sigma}^{(i)}(p)
\end{aligned}
$$

where $c_{m \sigma}^{(i) \dagger}(k)$ is the fermion creation operator with spin index $\sigma= \pm 1$, on the left (right) moving branches $(m=L / R)$ of the chain with index $i=1 / 2$, and $\epsilon_{R / L}(k)=v_{f}\left( \pm k-k_{f}\right)$ (see Figure 1 for clarification of labels). Neglecting all branch mixing 'backscattering' events, all four interactions in Figure 2 have been expressed above in terms of electronic densities $\rho_{m \sigma}^{(i)}(q)$. The $q=0$ component of the interaction terms, which contains information about chemical potential in the form of a bare charge $N_{m 0 \sigma}^{(i)}$, has been explicitly included. This will be seen to be important in what follows. We have also made the nonrestrictive assumption of a spin independent interaction so that $g^{\uparrow \uparrow}=g^{\uparrow \downarrow}=g$. This may easily be relaxed.

To illustrate more clearly the fact that the spectral properties of an isolated chain are not always an accurate guide to the coherence of interchain hopping in a two chain model, we will consider below a special soluble point of the interacting problem. In the interests of 
clarity we will suppress spin dependence. We will look at the issue of spin-charge separation later. (In fact the generalization of the model to include spin is straightforward, and does not affect any of the arguments of this section, which relate chiefly to the anomalous exponent $\alpha$.

If we rewrite the momentum-space Hamiltonian in terms of collective coordinates

$$
\begin{aligned}
\alpha^{\dagger}(q) & =\frac{1}{\sqrt{2}} \frac{2 \pi}{q L}\left[\rho_{R}^{(b)}(q)+\rho_{R}^{(a)}(q)\right] \\
\beta^{\dagger}(q) & =\frac{1}{\sqrt{2}} \frac{2 \pi}{q L}\left[\rho_{L}^{(b)}(-q)+\rho_{L}^{(a)}(-q)\right] \\
\gamma^{\dagger}(q) & =\frac{1}{\sqrt{2}} \frac{2 \pi}{q L}\left[\rho_{R}^{(b)}(q)-\rho_{R}^{(a)}(q)\right] \\
\delta^{\dagger}(q) & =\frac{1}{\sqrt{2}} \frac{2 \pi}{q L}\left[\rho_{L}^{(b)}(q)-\rho_{L}^{(a)}(q)\right]
\end{aligned}
$$

with $\left[\alpha(q), \alpha^{\dagger}(q)\right]=\delta_{q q^{\prime}},[\alpha, \beta]=0$ etc., we find that the kinetic energy and interaction parts of the Hamiltonian take on a particularly simple form, provided that we impose the restriction $g_{4 \|}=g_{4 \perp}=g_{4}, g_{2 \|}=g_{2 \perp}=g_{2}$. Then :

$$
\begin{aligned}
H_{0} & =\sum_{k \sigma} v_{f} q\left[\alpha^{\dagger}(q) \alpha(q)+\beta^{\dagger}(q) \beta(q)+\gamma^{\dagger}(q) \gamma(q)+\delta^{\dagger}(q) \delta(q)\right] \\
& +\frac{\pi v_{f}}{L}\left[\left(N_{\alpha}-N_{\alpha}^{0}\right)^{2}+\left(N_{\beta}-N_{\beta}^{0}\right)^{2}+\left(N_{\gamma}-N_{\gamma}^{0}\right)^{2}+\left(N_{\delta}-N_{\delta}^{0}\right)^{2}\right]
\end{aligned}
$$

and

$$
\begin{aligned}
V & =\sum_{q>0} \frac{g_{4} q}{L}\left[\alpha^{\dagger}(q) \alpha(q)+\beta^{\dagger}(q) \beta(q)\right] \\
& +\sum_{q>0} \frac{g_{2} q}{L}\left[\alpha^{\dagger}(q) \beta^{\dagger}(-q)+\alpha(-q) \beta(q)\right] \\
& +\frac{g_{4} q}{L}\left[\left(N_{\alpha}-N_{\alpha}^{0}\right)^{2}+\left(N_{\beta}-N_{\beta}^{0}\right)^{2}\right] \\
& +\frac{2 g_{2} q}{L}\left[\left(N_{\alpha}-N_{\alpha}^{0}\right)\left(N_{\beta}-N_{\beta}^{0}\right)\right]
\end{aligned}
$$

with $N_{\alpha}^{0}=N_{\beta}^{0}=\sqrt{2} N^{0}$ and $N_{\gamma}^{0}=N_{\delta}^{0}=\frac{t_{\perp} L}{\sqrt{2} \pi v_{f}}$.

Physically this choice of interaction corresponds to having equally strong interchain and intrachain interaction, at least for the small $q$ regime of the bosonization. While this condition is unlikely to be met in the majority of quasi-one dimensional conductors, we note in 
passing that a number of 'ladder' compounds, with nearly isotropic coupling between well spaced pairs of one-dimensional chains have recently been synthesized [34].

The Hamiltonian for the spinless two chain system above is then quadratic, and describes two independent one dimensional systems. It may be solved directly by canonical transformation. We can therefore proceed to calculate the correlation functions for the particles in binding and antibinding bands, using the methods standard in the bosonization literature. The correct non-interacting (retarded) Greens function can be correctly recovered from the bosonic expression for $H_{0}$ using [35] to give

$$
<\psi_{1 R}^{\dagger}(x, t) \psi_{1 R}(0,0)>_{0}=\frac{i}{2 \pi} \frac{\exp -i k_{f} x}{x-v_{f} t+i \epsilon} \cos \left[\frac{t_{\perp} x}{v_{f}}\right],
$$

demonstrating the continuity between the collective bosonic and single-particle fermionic descriptions at the noninteracting point. The effect of interchain hopping on free electrons is to introduce a cosine modulation in the single particle correlation function on a single chain reflecting the Fermi surface shifts associated with the formation of binding and antibinding states. This vanishes continuously as $t_{\perp} \rightarrow 0$. We may find the correlation function for our spinless interacting model by direct analogy with careful treatment of the one chain spinful case [2]

$$
\begin{aligned}
<\psi_{1 R}^{\dagger}(x, t) \psi_{1 R}(0,0)>= & \frac{i}{2 \pi} \exp \left(-i k_{f} x\right) \frac{\Lambda+i\left(v_{f} t-x\right)}{\epsilon+i\left(v_{f} t-x\right)} \\
& \frac{1}{\left[x-v_{\alpha} t+i \epsilon\right]^{\frac{1}{2}}\left[x-v_{f} t+i \epsilon\right]^{\frac{1}{2}}}\left(\frac{\Lambda^{2}}{\left(\Lambda+i v_{\alpha} t\right)^{2}+x^{2}}\right)^{\alpha / 2} \cos \left[\frac{t_{\perp} x}{v_{f}}\right],
\end{aligned}
$$

where $\alpha=\left[1-\left(g_{2} /\left(g_{4}+\pi v_{F}\right)\right)^{2}\right]^{-1 / 2}-1$ and $v_{\alpha}=\left[\left(v_{F}+g_{4} / \pi\right)^{2}-\left(g_{2} / \pi\right)^{2}\right]^{1 / 2} . \Lambda$ is a length scale set by the range of interaction. The effects of interaction on the model are apparent in the branch cut (analogous to "spin/charge" separation $v_{\alpha} \neq v_{f}$ ), and in the anomalous scaling $G(s x, s t)=s^{-1-\alpha} G(x, t)$. The eigenstates of this spinless coupled two chain model are clearly bosonic, and it is possible to show explicitly that the interacting ground state is orthogonal to the noninteracting ground state. We wish to emphasize that the same results are obtained in the limit $t_{\perp} \rightarrow 0$ as in the absence of $t_{\perp}$, and to remark that this model may be mapped onto the case of a single chain of spinful electrons in a magnetic field. 
We also learn from this correlation function that the Fermi surface shifts have survived the introduction of interaction, despite the consequent Luttinger Liquid character of motion along the chains. The momentum distribution $n(k)$ will have power law behavior $n\left(k-k_{f}\right) \sim$ $\left(k-k_{f}\right)^{\alpha}$ in both the binding and antibinding bands, but with different values of $k_{f}$ in each band.

This is strongly suggestive of coherent electronic motion between the chains, and in fact for our model with the special choice $g_{2 \perp}=g_{2 \|}=g_{2}, g_{4 \perp}=g_{4 \|}=g_{4}$,

$$
\left[H_{0 \perp}, V_{\|}\right]=-\left[H_{0 \perp}, V_{\perp}\right]
$$

and the quantity $P(t)$ (1) behaves exactly as in a free electron gas. This is despite the fact that the single particle correlation function does not possess a pole at the Fermi momentum, and that the interacting groundstate is orthogonal to the non interacting ground state. We understand from the commutator above that the source of any incoherence of hopping between chains in a more general two chain model are those non-branch conserving terms in the Hamiltonian which render the model insoluble by bosonization, and which give rise to the rich variety of dominant fluctuations found by scaling arguments.

An alternative physically motivated test for the coherence of hopping between the chains is provided by the interchain polarization response function, whose associated spectral function is given by

$$
\rho_{\Delta N}(w)=\int_{0}^{\infty} d t e^{-i \omega t} \Im<[\Delta N(t), \Delta N(0)]>
$$

where the operator $\Delta N=N_{1}-N_{2}$ is the difference between the number operators for a given branch of the two chains. For free electrons with linear dispersion this may easily be shown to be monochromatic - the only frequency entering the response is the binding/antibinding energy gap $2 t_{\perp}$. We may recover the same result from the bosonic form of $H_{0}$ by calculating $\left[H_{0},\left[H_{0}, \Delta N\right]\right]$ to show

$$
\frac{\partial^{2} \Delta N}{\partial t^{2}}=-\left(2 t_{\perp}\right)^{2} \Delta N
$$


reflecting the fact that $\Delta N$ is not a good quantum number in the presence of hopping. This commutator would vanish, in the both interacting and the free electron case, were the chemical potential terms in the Hamiltonian to be neglected. The finite expectation value of $(\Delta N)^{2} \sim 2 t_{\perp}$ in the noninteracting ground state may then be understood in terms of the zero point motion of a simple harmonic oscillator with $\Delta N$ as its coordinate, and canonical momentum proportional to the current operator $c_{1}^{\dagger} c_{2}-c_{2}^{\dagger} c_{1}$. The raising operator for this oscillator is in turn proportional to $N_{\gamma}$ (for the right moving branch, see 18), with $<N_{b}+N_{a}>=<N_{1}+N_{2}>$ a constant of motion with or without $t_{\perp}=0$. The inclusion of our special interaction does not change this result; again we find a monochromatic polarization response function and $\Delta N^{2} \sim 2 t_{\perp}$, confirming the coherent nature of the hopping.

We see that it is possible to propose a model for a two chain system which is a Luttinger Liquid in the chain direction for any finite $g_{2}$, and to show directly from the Hamiltonian, from the correlation functions, or from the response of the system to interchain polarization, that it displays fully coherent hopping between the chains, with associated shifts in the "Fermi-surface" for any finite $t_{\perp}$. These two properties are thus found in this special case to be entirely independent of one another; anomalous scaling in the chain direction coexists with coherent hopping in the interchain direction. The intuitive idea that the spectral properties of the model for $t_{\perp}=0$ determine the possibility of confinement at finite $t_{\perp}$ is seen in this instance to fail completely.

We may generalize those arguments which relate to the commutation relations of the hopping term to $\mathrm{N}$ chains simply by including a nearest neighbor interchain interaction between each pair of chains to cancel the undesired commutator $\left[V_{\|}, H_{0 \perp}\right]$. However the generalization of the bosonization scheme to the evaluation of correlators for the N-chain case is not quite so straightforward. Thus while it seems to be possible to achieve coherent interchain transport in a two chain model without the formation of a pole in the correlation function, it is not clear that we may make the same statement about an $\mathrm{N}$ chain model. Our two chain model is also insensitive to the size of $t_{\perp}$, but should not be considered physical for any value of $t_{\perp}$ bigger than a scale set by the curvature of the real free fermion dispersion 
and the interaction range $\Lambda$ ).

\section{COHERENCE OF HOPPING AND SPIN CHARGE SEPARATION}

Spin charge separation is perhaps the best known of the unusual properties of the one dimensional interacting electron gas. In the Luttinger model (for which the Luttinger Liquid ground state is an exact solution), it has its origin in the fact that the elementary excitations of the gas are not fermionic quasiparticles, but rather bosonic collective modes. In the presence of a spin-dependent interaction (such as an exchange potential), the modes associated with spin and charge degrees of freedom need not disperse with the same velocity.

A hopping term of the form of $H_{0 \perp}$, however, must transfer a 'real' electron, with a corresponding non-trivial adjustment in the spin and charge excitations on each chain. Naively we might expect this to frustrate hopping between chains, and so protect (at least partially) the one-dimensional interacting Fermi gas from the destabilizing effects of higher dimension. Even if spin charge separation alone is not enough to generate confinement of the electrons on a single chain, it might be expected to reduce the coherence of hopping between chains and, by reducing the 'phase memory' of the transferred electron, to hinder the formation of band structure perpendicular to the chains. In this section, we will consider a soluble one branch, spinful, two-chain model with inter- and intra-chain $g_{4}$ interaction, and assess the effect of the spin-charge separation which it exhibits on the coherence of hopping between chains.

We may solve the one branch model of an electron gas with linear dispersion and forward scattering interactions by bosonization :

$$
H_{1}=\sum_{k \sigma} v_{f}\left(k-k_{f}\right) c_{\sigma}^{\dagger(1)}(k) c_{\sigma}^{(1)}(k)+\frac{1}{2 L} \sum_{q \sigma \sigma^{\prime}}\left(g_{4 \|}^{\uparrow \uparrow} \delta_{\sigma \sigma^{\prime}}+g_{4 \|}^{\uparrow \downarrow} \delta_{\sigma-\sigma^{\prime}}\right) \rho_{\sigma}^{(1)}(q) \rho_{\sigma^{\prime}}^{(1)}(-q)
$$

where $\rho_{\sigma}^{(1)}(q)$ is the Fourier transform of the density operator for electrons with spin index $\sigma$ on the chain with index 1 . In fact it is possible also to solve exactly the two chain problem

$$
H=H_{1}+H_{2}+H_{0 \perp}
$$




$$
H_{0 \perp}=-t_{\perp} \sum_{k \sigma} c_{\sigma}^{\dagger(1)}(k) c_{\sigma}^{(2)}(k)+c_{\sigma}^{\dagger(2)}(k) c_{\sigma}^{(1)}(k)
$$

by expressing $H_{0 \perp}$ in terms of the bosonic representation of the electronic field operators on each chain, as illustrated by Fabrizio and Parola [25,26]. The Hamiltonian can then be expressed in a basis comprising four branches of spinless fermions with linear dispersion

$$
\begin{gathered}
H_{1}+H_{2} \rightarrow \sum_{k>0} u_{\rho} k\left[a_{\rho}^{\dagger}(k) a_{\rho}(k)+b_{\rho}^{\dagger}(k) b_{\rho}(k)\right]+u_{\sigma} k\left[a_{\sigma}^{\dagger}(k) a_{\sigma}(k)+b_{\sigma}^{\dagger}(k) b_{\sigma}(k)\right] \\
H_{0 \perp} \rightarrow-2 t_{\perp} \sum_{k>0} b_{\rho}^{\dagger}(k) b_{\sigma}(k)+b_{\sigma}^{\dagger}(k) b_{\rho}(k) .
\end{gathered}
$$

where,

$$
\begin{aligned}
& u_{\rho}=v_{f}+\frac{\left(g_{4 \|}^{\uparrow \uparrow}+g_{4 \|}^{\uparrow \downarrow}\right)}{2 \pi} \\
& u_{\sigma}=v_{f}+\frac{\left(g_{4 \uparrow}^{\uparrow \uparrow}-g_{4 \|}^{\uparrow \downarrow}\right)}{2 \pi} .
\end{aligned}
$$

This may be diagonalized by canonical transformation to yield four branchs of free, spinless, fermions with dispersion

$$
\begin{aligned}
\epsilon_{\rho}(k) & =u_{\rho} k \\
\epsilon_{\sigma}(k) & =u_{\sigma} k \\
\epsilon_{ \pm}(k) & =\frac{1}{2}\left(u_{\rho}+u_{\sigma}\right) k \pm \sqrt{\left[\frac{1}{2}\left(u_{\rho}-u_{\sigma}\right)^{2}+4 t_{\perp}^{2}\right.},
\end{aligned}
$$

and the ground state found by filling the fermion branch with dispersion $\epsilon_{-}(k)$ up to the chemical potential $\mu=0$. The expectation value of the number operator $N^{(b)}-N^{(a)}$ can be calculated and is strongly suggestive of the formation of 'band structure' in the interchain direction :

$$
\frac{<N^{(b)}-N^{(a)}>}{L}=\frac{1}{u_{\rho}-u_{\sigma}} \ln \left(\frac{u_{\rho}}{u_{\sigma}}\right) \frac{4 t_{\perp}}{2 \pi} .
$$

In fact the commutator $\left[H_{1}+H_{2}, H_{\perp}\right]$ does not vanish, so $N^{(b)}-N^{(a)}$ is not a good quantum number for the system, but it is clear that the splitting of energy levels due to $t_{\perp}$ is only partially suppressed. Those terms in the Hamiltonian which do not commute with $N^{(b)}-N^{(a)}$ give rise to the prefactor 


$$
\frac{1}{u_{\rho}-u_{\sigma}} \ln \left(\frac{u_{\rho}}{u_{\sigma}}\right) \leq 1
$$

which would be unity in the free electron case. It is a consequence of the curvature in $\epsilon_{-}(k)$, which depends on both $t_{\perp}$ and $u_{\rho}-u_{\sigma}$, and indicates a suppression of dispersion in the interchain direction in the presence of spin charge separation $u_{\rho} \neq u_{\sigma}$. As such it may be taken as a measure of the incoherence of hopping between chains.

It is not difficult to generalize the model of Fabrizio and Parola to include interchain interaction; the structure of the Hamiltonian is altered only in that the $a(k)$ and $b(k)$ fermions acquire different velocities

$$
H_{1}+H_{2} \rightarrow \sum_{k>0}\left[u_{\rho a} k a_{\rho}^{\dagger}(k) a_{\rho}(k)+u_{\rho b} k b_{\rho}^{\dagger}(k) b_{\rho}(k)+u_{\sigma a} k a_{\sigma}^{\dagger}(k) a_{\sigma}(k)+u_{\sigma b} k b_{\sigma}^{\dagger}(k) b_{\sigma}(k)\right],
$$

where,

$$
\begin{aligned}
& u_{\rho a}=v_{f}+\frac{\left(g_{4 \|}^{\uparrow \uparrow}+g_{4 \|}^{\uparrow \downarrow}+g_{4 \perp}^{\uparrow \uparrow}+g_{4 \perp}^{\uparrow \downarrow}\right)}{2 \pi} \\
& u_{\rho b}=v_{f}+\frac{\left(g_{4 \|}^{\uparrow \uparrow}+g_{4 \|}^{\uparrow \uparrow}-g_{4 \perp}^{\uparrow \uparrow}-g_{4 \perp}^{\uparrow \downarrow}\right)}{2 \pi} \\
& u_{\sigma a}=v_{f}+\frac{\left(g_{4 \|}^{\uparrow \uparrow}-g_{4 \|}^{\uparrow \downarrow}+g_{4 \perp}^{\uparrow \uparrow}+g_{4 \perp}^{\uparrow \downarrow}\right)}{2 \pi} \\
& u_{\sigma b}=v_{f}+\frac{\left(g_{4 \|}^{\uparrow \uparrow}-g_{4 \|}^{\uparrow \downarrow}-g_{4 \perp}^{\uparrow \uparrow}+g_{4 \perp}^{\uparrow \downarrow}\right)}{2 \pi} .
\end{aligned}
$$

The dispersion of the four fermionic branches after diagonalization are thus modified to give

$$
\begin{aligned}
\epsilon_{\rho}(k) & =u_{\rho a} k \\
\epsilon_{\sigma}(k) & =u_{\sigma a} k \\
\epsilon_{ \pm}(k) & =\frac{1}{2}\left(u_{\rho b}+u_{\sigma b}\right) k \pm \sqrt{\left[\frac{1}{2}\left(u_{\rho b}-u_{\sigma b}\right)^{2}+4 t_{\perp}^{2}\right.}
\end{aligned}
$$

The ground state expectation value of $N^{(b)}-N^{(a)}$ is then given by the same formula as before (33) but with $u_{\rho}$ and $u_{\sigma}$ replaced by $u_{\rho b}$ and $u_{\sigma b}$. There is full coherence (the prefactor (34) equal to one) when the dispersion $\epsilon_{ \pm}(k)$ is linear in $k$. This happens not just when all $g_{4}$ 's are equal to zero (the non-interacting case) but also when $g_{4 \|}^{\uparrow \downarrow}=g_{4 \perp}^{\uparrow}$. In either case 
$\left[H_{1}+H_{2}, H_{\perp}\right]=0$. In the special case of equal inter and intrachain interactions, we may solve the model directly by bosonization, obtaining the same ground state and correlators as are found by refermionization :

$$
<\psi_{0}^{\dagger}(x, t) \psi_{0}(0,0)>\sim e^{i\left(k_{f}+\Delta k_{f}\right) x}\left(x-v_{f} t+i \epsilon\right)^{-1 / 2}\left(x-u_{\rho b} t+i \epsilon\right)^{-1 / 4}\left(x-u_{\sigma b} t+i \epsilon\right)^{-1 / 4},
$$

where $\Delta k_{f}=\frac{t_{\perp}}{v_{f}}$.

In the general case, the prefactor (34) is less than one and depends on the relative strength of the interchain and interchain interactions. This result is consistent with the arguments of CSA [20] who also consider the model studied by Fabrizio and Parola. They find evidence of macroscopic coherence for times much less than $\left[\frac{2 \pi \Delta N}{L}\left(u_{\rho}-u_{\sigma}\right)\right]^{-1}[37$.

\section{CONCLUSIONS}

We have shown that it is possible for a two-chain system to show both coherent hopping between chains and the anomalous intrachain scaling characteristic of a Luttinger liquid. For the two chain Luttinger model, which we can solve exactly for a special choice of interchain interaction, we do not find any evidence for the formation of a pole at the Fermi surface. In general, we find that the degree of coherence observed in the interchain hopping depends rather sensitively on the precise details of the interactions both on the chains and between the chains and may not always be estimated from the spectral properties of the individual chains alone.

A simple one-branch model exhibiting spin-charge separation is found to be neither completely coherent nor completely incoherent in its macroscopic evolution, and we draw the conclusion, in line with other authors, that spin charge separation alone may suppress, but is not sufficient to prevent the formation of band structure in the hopping direction. Again, for a special choice of interchain interaction it is possible to arrive at a model which displays both spin charge separation and completely coherent hopping between chains.

Although the restriction on interaction imposed by the criterion for coherence we have used is rather severe, and gives evidence of the stability of a Luttinger Liquid ground state 
only on the line (plane, in spinful case) $g_{\perp}=g_{\|}$, the states found away from this line (plane) are known from scaling arguments to be ordered. We speculate that at temperatures greater than a scale set by the gap of each ordered phase, the behavior of the system will be controlled by this $g_{\perp}=g_{\|}$(quantum critical) line.

Nic Shannon wishes to acknowledge helpful conversations with David Clarke, Andrey Chubakov, George Rowlands, Edmound Orignac, Carol Hayward, Sylvan Capponi and Thanassis Yannacopoulis, and also an HCM grant for attendance at the ISI Workshop on Strong Correlation in Low Dimensional Systems held in May 1996. 


\section{REFERENCES}

[1] V. J. Emery, in "Highly Conducting One-dimensional Solids", Plenum, N. Y. (1979)

[2] J. Voit, Rep. Prog. Phys. 57, 977, (1994)

[3] see, e.g. A. Houghton and J. B. Marston, Phys. Rev. B 48, 7790 (1993); A. Houghton, H. J. Kwon, and J. B. Marston, ibid, 50, 1351 (1994); A. H. Castro Neto and E. Fradkin, Phys. Rev. Lett. 72, 1393 (1994); Phys. Rev. B 49, 10877 (1994); Y. M. Li, ibid, 51, 13046 (1995); P. Kopietz and K. Schonhammer, Z. Physik B 100, 259 (1996).

[4] See, e.g., J. R. Engelbrecht and M. Randeria, Phys. Rev. Lett. 65, 1032 (1990); 66, 3325 (1991); Phys. Rev. B 45, 12419 (1992); C. Di Castro, C. Castellani, and W. Metzner, Phys. Rev. Lett. 72, 316 (1994); R. Shankar, Rev. Mod. Phys. 66, 129 (1994). M. Fabrizio, A. Parola, and E. Tosatti, Phys. Rev. B 44, 1033 (1991); H. Fukuyama, O. Narikiyo, and Y. Hasegawa, J. Phys. Soc. Jpn 60, 372 (1991).

[5] A. M. Finkelstein and A. I. Larkin, Phys. Rev. B. 47, 10461, (1993)

[6] P. Koepitz, V. Meden and K. Schönhammer, Phys. Rev. Lett. 74, 2997, (1995)

[7] A. A. Nersesyan, Phys. Lett. A 153, 49, (1991).

[8] A. Nersesyan, A. Luther and F. Kusmartsev, Phys. Lett. A 176, 363, (1993)

[9] T. Giamarchi and H. J. Schultz, J. Phys. France 49, 819, (1988)

[10] E. Orignac and T. Giamarchi, Phys. Rev. B 53, 10453, (1996)

[11] C. A. Hayward and D. Poilblanc, Phys. Rev. B 53, 11721, (1996); C. A. Hayward, D. Poilblanc and D. J. Scalapino, ibid 53, R8863, (1996); C. A. Hayward, D. Poilblanc, R. M. Noack, D. J. Scalapino, W. Hanke, Phys. Rev. Lett. 75, 926, (1995)

[12] H. Enders, R. M. Noack, W. Hanke, D. poilblanc, D. J. Scalapino, Phys. Rev. B 53, 5530, (1996); R. M. Noack, S. R. White, D. J. Scalapino, Europhys. Lett. 30, 163, 
(1995); S. R. White, R. M. Noack and D. J. Scalapino, J. Low Temp. Phys. 99, 593, (1995); R. M. Noack, S. R. White and D. J. Scalapino, Phys. Rev. Lett 73, 882, (1994)

[13] D. Boies, C. Bourbonnais and A.M.S. Tremblay, (1996) SISSA cond-mat 9604122.

[14] C. Bourbonnais and L. G. Caron, Int. J. Mod. Phys. B 5, 1033, (1991)

[15] X. G. Wen, Phys. Rev. B 42, 6623, (1990)

[16] S. Tomonaga, Prog. Theor. Phys. 5, 544, (1950)

[17] F. D. M. Haldane, J. Phys. C 14, 2585, (1981)

[18] A. Luther and V. J. Emery, Phys. Rev. Lett. 33, 589, (1974)

[19] P. W. Anderson, Phys. Rev. Lett. 67, 3844, (1991)

[20] D. G Clarke, S. P. Strong and P. W. Anderson, Phys. Rev. Lett. 72, 3218, (1993)

[21] S. P. Strong, D. G Clarke and P. W. Anderson, Phys. Rev. Lett. 73, 1007, (1994)

[22] D. G Clarke, S. P. Strong and P. W. Anderson, Phys. Rev. Lett. 74, 4499, (1995)

[23] S. Chakravarty, A. Sudbo, P. W. Anderson, and S. Strong, Science, 261, 337 (1993).

[24] A. Tsvelik, preprint cond-mat/9607209 v2 (1996)

[25] M. Fabrizio and A. Parola, Phys. Rev. Lett. 70, 226, (1993)

[26] M. Fabrizio, Phys. Rev. B. 48, 15838, (1993)

[27] S. Capponi, D. Poilblanc and F. Mila, preprint cond-mat/9608004 (1996)

[28] A. Tsvelik, unpublished work.

[29] S. Chakravarty and J. Rudnick, Phys. Rev. Lett. 75, 501, (1995)

[30] S. Chakravarty and P. Anderson, Phys. Rev. Lett. 72, 3859, (1994)

[31] This is in fact not quite correct. If we explicitly include the shift in the value of $k_{f}$ 
required to restore the chemical potential to zero on the chain with additional charge $\Delta N$, we find eigenvalues $\epsilon(k)=v_{f}\left(k-k_{f}\right)+\frac{\Delta \mu}{2} \pm \sqrt{t_{\perp}^{2}+\Delta \mu^{2}}$ where $\Delta \mu=\frac{2 \pi v_{f}}{L} \Delta N$, corresponding to a slightly different rotation of coordinates from the binding/antibinding transformation we shall use, and modifying the frequency of oscillation between levels from $t_{\perp}$ to $t_{\perp}^{*}=\sqrt{t_{\perp}^{2}+\Delta \mu^{2}}$. This is a small quantitative effect, and modifies our results only in the correction $t_{\perp} \rightarrow t_{\perp}^{*}$, so we shall neglect it in what follows.

[32] This is essentially the phenomenon known as Landau damping in plasma physics. Where it is possible to take the limit of a continuous smooth distribution of frequencies $\sum_{n}\left|c_{n}\right|^{2} \rightarrow \int d \omega d(\omega)$, the degree of damping exhibited depends on the structure of the distribution $d(\omega)$, with greater damping exhibited for distributions over a broad range of $\omega$ [32. See eg D. E. Baldwin and G. Rowlands, Phys. Fluids 9, 2444, (1966)

[33] F. Mila and D. Poilblanc, Phys. Rev. Lett. 76, 287, (1996)

[34] E. Dagotto and T. M. Rice, Science 271, 618, (1996)

[35] J. Solyom, Adv. Phys. 28, 201 (1979); V. J. Emery, Highly Conducting One-dimensional Solids (Plenum NY, 1979), p247.

[36] M. Fabrizio, A. Parola and E. Tosatti, Phys. Rev. B 46, 3159, (1992)

[37] This time-scale can also be obtained by consideration of the failure of $H_{1}+H_{2}$ and $H_{\perp}$ to commute, N. Shannon 1996, unpublished. 


\section{FIGURES}

FIG. 1. Dispersion relations for the four branches of the two chain Luttinger model, showing interchain hopping $t_{\perp}$.

FIG. 2. Examples of chain and branch indices for the four interaction terms kept in the bosonic treatment of the two chain problem. 